\title{
Column Rank Distances of Rank Metric Convolutional Codes
}

\author{
Diego Napp ${ }^{\star}$, Raquel Pinto ${ }^{\star}$ Joachim Rosenthal $^{\S}$ and Filipa Santana ${ }^{\star}$ \\ diego@ua.pt, raquel@ua.pt, rosenthal@math.uzh.ch, vfssantana@ua.pt \\ * CIDMA - Center for Research and Development in Mathematics and Applications, \\ Department of Mathematics, University of Aveiro, Portugal \\ $\S$ Department of Mathematics, University of Zurich, \\ Winterthurstrasse 190, CH-8057 Zürich, Switzerland
}

\begin{abstract}
In this paper, we deal with the so-called multi-shot network coding, meaning that the network is used several times (shots) to propagate the information. The framework we present is slightly more general than the one which can be found in the literature. We study and introduce the notion of column rank distance of rank metric convolutional codes for any given rate and finite field. Within this new framework we generalize previous results on column distances of Hamming and rank metric convolutional codes $[3,8]$. This contribution can be considered as a continuation follow-up of the work presented in [11].
\end{abstract}

\section{Introduction}

The theory of Random Linear Network Coding has been mainly devoted to noncoherent one-shot network coding, meaning that the random structure of the network is used just once to propagate information. One of the problems in this situation is that in order to increase the error-correcting capabilities of a code, one necessarily needs to increase the field size or the packet size and this might not be optimal or impossible in many applications.

Hence, in these situations one of the solutions proposed is to create dependencies across multiple shots aiming to approach the channel capacity. In fact, it was been recently shown that spreading redundancy among the transmitted codewords (row spaces) at different instances (shots) can improve the error-correction capabilities of the code. These ideas gave rise to the area of multi-shot network coding. Although the potential of using multi-shot network coding was already observed in the seminal paper [5], only recently this interesting approach has been investigated $[1,7,15,17]$.

There are basically two ways for constructing multi-shot codes: one using concatenation of codes and other using rank metric convolutional codes. In [15],

\footnotetext{
* This work was supported by Portuguese funds through the Center for Research and Development in Mathematics and Applications (CIDMA), and The Portuguese Foundation for Science and Technology (FCT - Fundação para a Ciência e a Tecnologia), within project UID/MAT/04106/2013.
} 
a concatenated code was introduced based on a multilevel code construction. In [13], a concatenation scheme was presented using a Hamming metric convolutional code as an outer code and a rank metric code as an inner code. A different type of concatenation was introduced in [7] where the authors use codes that layer both Maximum Sum Rank (MSR) codes and Gabidulin in order to achieve the streaming capacity for the Burst Erasure Channel.

Apart from concatenated codes, another very natural way to spread redundancy across codewords is by means of convolutional codes [2-4, 9, 14]. Adapting this class of codes to the context of networks brought about the notion of rank metric convolutional codes and interestingly there has been little research on these codes, see $[1,6-8,17]$. The work in [17] was pioneer in this direction by presenting the first class of rank metric convolutional codes together with a decoding algorithm able to deal with errors, erasures and deviations. However, the results were only valid for unit memory convolutional codes and in $[1,6-8]$ (see also the references therein) an interesting and more general class of rank metric convolutional codes was introduced to cope with network streaming applications.

In this paper we continue our work in [11] and propose a framework slightly more general than the existing ones in the literature on rank metric convolutional codes. In the proposed framework, rank metric codes can be defined for all rates and fields. In this setting, an extension of the standard rank metric has been considered to provide the proper measure for the number of rank erasures that a multi-shot network code can tolerate. Here we continue this line of work and investigate the notion of column rank distance of rank metric convolutional codes in this more general setting. We show that the existing results on column distance in both contexts of Hamming [3] and rank metric [8] can be generalized to this more general point of view.

\section{Convolutional Codes}

Let $\mathbb{F}$ be a finite field and $\mathbb{F}[D]$ be the ring of polynomials with coefficients in $\mathbb{F}$. A convolutional code $C$ of rate $k / n$ is an $\mathbb{F}[D]$-submodule of $\mathbb{F}[D]^{n}$, with rank $k$. If $G(D) \in \mathbb{F}[D]^{k \times n}$ is a full row rank matrix such that

$$
\mathcal{C}=\operatorname{im}_{\mathbb{F}_{q}[D]} G(D)=\left\{u(D) G(D): u(D) \in \mathbb{F}[D]^{k}\right\},
$$

then $G(D)$ is called an encoder of $C$.

Any other encoder $\tilde{G}(D)$ of $\mathcal{C}$ differ from $G(D)$ by left multiplication by a unimodular matrix $U(D) \in \mathbb{F}[D]^{k \times k}$, i.e., $\tilde{G}(D)=U(D) G(D)$. Therefore, if $\mathcal{C}$ admits a left prime convolutional encoder then all its encoders are left prime. Such a code is called observable.

A convolutional code always admits a minimal encoder, i.e., in row reduced form. ${ }^{1}$ The sum of the row degrees of a minimal encoder attains its minimum

\footnotetext{
${ }^{1}$ A polynomial matrix $G(D) \in \mathbb{F}[D]^{k \times n}$ is in row reduced form if the constant matrix $G_{l r c}$, called leading row coefficient matrix, constituted by the coefficients of the term of degree equal to the row degree, is full row rank.
} 
among all the encoders of $\mathcal{C}$. Such sum is usually denoted by $\delta$ and called the degree of $\mathcal{C}$. A rate $k / n$ convolutional code $\mathcal{C}$ of degree $\delta$ is called an $(n, k, \delta)$ convolutional code [10].

The free distance and the column distances of a convolutional code are important measures of the capability of error detection and error correction of the code. The free distance of a convolutional code $\mathcal{C}$ is given by

$$
d_{\text {free }}(\mathcal{C})=\min _{v(D) \in \mathcal{C}, v(D) \neq 0} w t(v(D))
$$

where $w t(v(D))$ is the Hamming weight of a polynomial vector

$$
v(D)=\sum_{i \in \mathbb{N}_{0}} v_{i} D^{i} \in \mathbb{F}[D]^{n}
$$

defined as

$$
w t(v(D))=\sum_{i \in \mathbb{N}_{0}} w t\left(v_{i}\right)
$$

being $w t\left(v_{i}\right)$ the number of the nonzero components of $v_{i}$.

Rosenthal and Smarandache[16] showed that the free distance of an $(n, k, \delta)$ convolutional code is upper bounded by

$$
d_{\text {free }}(\mathcal{C}) \leq(n-k)\left(\left\lfloor\frac{\delta}{k}\right\rfloor+1\right)+\delta+1 .
$$

This bound was called the generalized Singleton bound. An $(n, k, \delta)$ convolutional code whose free distance is equal to the generalized Singleton bound is called maximum distance separable (MDS) code [16].

Let us now consider the column distances of an $(n, k, \delta)$ convolutional code $\mathcal{C}$. For that we will consider that $\mathcal{C}$ is observable. Observable convolutional codes admit a kernel representation $H(D) \in \mathbb{F}[D]^{(n-k) \times n}$, i.e. such that $\mathcal{C}=\operatorname{ker} H(D)$. Let

$$
G(D)=\sum_{j=0}^{\nu} G_{j} D^{j} \in \mathbb{F}[D]^{k \times n}, G_{i} \in \mathbb{F}^{k \times n}, G_{\nu} \neq 0
$$

be an encoder of $\mathcal{C}$ and

$$
H(D)=\sum_{j=0}^{\mu} H_{j} D^{j} \in \mathbb{F}[D]^{(n-k) \times n}, H_{i} \in \mathbb{F}^{(n-k) \times n}, H_{\mu} \neq 0
$$

be a parity-check matrix of $\mathcal{C}$. For every $j \in \mathbb{N}_{0}$, the truncated sliding generator matrices $G_{j}^{c} \in \mathbb{F}^{(j+1) k \times(j+1) n}$ and the truncated sliding parity-check matrices $H_{j}^{c} \in \mathbb{F}^{(j+1)(n-k) \times(j+1) n}$ are given by

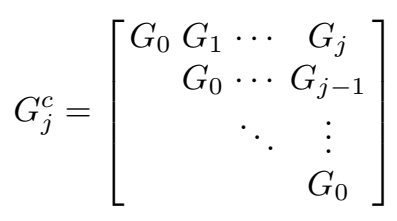




$$
H_{j}^{c}=\left[\begin{array}{cccc}
H_{0} & & & \\
H_{1} & H_{0} & & \\
\vdots & \vdots & \ddots & \\
H_{j} & H_{j-1} & \ldots & H_{0}
\end{array}\right],
$$

respectively, and when $j>\nu$, we let $G_{j}=0$ and when $j>\mu, H_{j}=0$.

Using the above assumptions the $j$-th column distance of $\mathcal{C}$ is given by

$$
\begin{aligned}
& d_{j}^{c}=\min \left\{w t\left((v(D))_{\mid[0, j]}\right): v_{0} \neq 0\right\} \\
& =\min \left\{w t\left(\left[v_{0} v_{1} \cdots v_{j}\right]\right):\left[v_{0} v_{1} \cdots v_{j}\right]=\left[u_{0} u_{1} \cdots u_{j}\right] G_{j}^{c}, u_{i} \in \mathbb{F}^{k}, u_{0} \neq 0\right\} \\
& =\min \left\{w t(v), v=\left(v_{0}, \ldots, v_{j}\right) \in \mathbb{F}^{(j+1) n}, v\left(H_{j}^{c}\right)^{T}=0, v_{0} \neq 0\right\},
\end{aligned}
$$

The following results give a bound on the column distances of an $(n, k, \delta)$ convolutional code and some properties of these distances.

Proposition 1 [3, Proposition 2.2]

Let $\mathcal{C}$ be an $(n, k, \delta)$ convolutional code. For every $j \in \mathbb{N}_{0}$ we have

$$
d_{j}^{c} \leq(n-k)(j+1)+1 .
$$

Corollary 2 [3, Corollary 2.3]

Let $\mathcal{C}$ be an $(n, k, \delta)$ convolutional code. If $d_{j}^{c}=(n-k)(j+1)+1$ then $d_{i}^{c}=$ $(n-k)(i+1)+1$, for every $i \leq j$.

Proposition 3 [3, Proposition 2.7]

Let $\mathcal{C}$ be an $\operatorname{MDS}(n, k, \delta)$ convolutional code with column distances $d_{j}^{c}, j \in \mathbb{N}_{0}$ and free distance $d_{\text {free }}$. Let $M=\min \left\{j \in \mathbb{N}_{0}, d_{j}^{c}=d_{\text {free }}\right\}$. Then,

$$
M \geq\left\lfloor\frac{\delta}{k}\right\rfloor+\left\lceil\frac{\delta}{n-k}\right\rceil .
$$

\section{Rank metric convolutional Codes}

In this section we will define rank metric convolutional codes whose codewords are polynomials matrices in $\mathbb{F}[D]^{n \times m}$ and we aim to further explore this more general approach in order to introduce the definition of column rank distances of a rank metric convolutional code and to propose a bound on this important measure.

A rank metric convolutional code $\mathcal{C} \subset \mathbb{F}^{n \times m}$ is the image of an homomorphism $\varphi: \mathbb{F}[D]^{k} \rightarrow \mathbb{F}[D]^{n \times m}$. We write $\varphi=\psi \circ \gamma$ as a composition of a monomorphism $\gamma$ and an isomorphism $\psi$ :

$$
\begin{gathered}
\varphi: \mathbb{F}[D]^{k} \stackrel{\gamma}{\longrightarrow} \mathbb{F}[D]^{n m} \stackrel{\psi}{\longrightarrow} \mathbb{F}[D]^{n \times m} \\
u(D) \mapsto v(D)=u(D) G(D) \mapsto V(D)
\end{gathered}
$$


where $G(D) \in \mathbb{F}^{k \times n m}$ is a full row rank polynomial matrix, called encoder of $\mathcal{C}$, and let $V(D)=\operatorname{rmat}_{n \times m}(v(D))$, such that $V_{i, j}(D)=v_{m i+j}(D)$, i.e., the rows of $V(D)$ are $n$ consecutive blocks with $m$ elements of $v(D)$.

As for convolutional codes, two encoders of $\mathcal{C}$ differ by left multiplication by a unimodular matrix and therefore $\mathcal{C}$ always admits minimal encoders. The degree $\delta$ of a rank metric convolutional code $\mathcal{C}$ is the sum of the row degrees of a minimal encoder of $\mathcal{C}$, i.e. the minimum value of the sum of the row degrees of its encoders. Rank metric convolutional codes with left prime encoders will also be called observable.

A rank metric convolutional code $\mathcal{C}$ of degree $\delta$, defined as in (1), is called an $(n \times m, k, \delta)$-rank metric convolutional code.

When dealing with rank metric codes a different measure of distance must be considered. The rank weight of a polynomial matrix $A(D)=\sum_{i \in \mathbb{N}_{0}} A_{i} D^{i} \in$ $\mathbb{F}[D]^{n \times m}$, is given by

$$
\operatorname{rwt}(A(D))=\sum_{i \in \mathbb{N}_{0}} \operatorname{rank} A_{i}
$$

If $B(D)=\sum_{i \in \mathbb{N}_{0}} B_{i} \in \mathbb{F}[D]^{n \times m}$, we define the sum rank distance between $A(D)$ and $B(D)$ as

$$
\begin{aligned}
d_{\mathrm{SR}}(A(D), B(D)) & =\operatorname{rwt}(A(D)-B(D)) \\
& =\sum_{i \in \mathbb{N}_{0}} \operatorname{rank}\left(A_{i}-B_{i}\right) .
\end{aligned}
$$

Lemma 4 The sum rank distance $d_{S R}$ is a distance in $\mathbb{F}[D]^{n \times m}$.

Next we will focus on two sum rank distances definitions of a rank metric convolutional code. The sum rank distance defined in $[12,11]$ and the novel notion of column rank distance.

The sum rank distance of a rank metric convolutional code $\mathcal{C}$ is defined as

$$
\begin{aligned}
d_{\mathrm{SR}}(\mathcal{C}) & =\min _{V(D), U(D) \in \mathcal{C}, V(D) \neq U(D)} d_{\mathrm{SR}}(V(D), U(D)) \\
& =\min _{0 \neq V(D) \in \mathcal{C}} \operatorname{rwt}(V(D)) .
\end{aligned}
$$

Next theorem establishes a bound on the sum rank distance of a rank metric convolutional code. Analogously as for the free distance of a convolutional code, this bound is referred as the Singleton bound for rank metric convolutional codes.

Theorem 5. [11, Theorem 3][12, Theorem 3] Let $\mathcal{C}$ be an $(n \times m, k, \delta)$ rank metric convolutional code. Then the sum rank distance of $\mathcal{C}$ is upper bounded by

$$
d_{S R}(\mathcal{C}) \leq n\left(\left\lfloor\frac{\delta}{k}\right\rfloor+1\right)-\left\lceil\frac{k\left(\left\lfloor\frac{\delta}{k}\right\rfloor+1\right)-\delta}{m}\right\rceil+1 .
$$

An $(n \times m, k, \delta)$ rank metric convolutional code whose sum rank distance attains the Singleton bound is called Maximum Rank Distance (MRD).

Let us now restrict to $(n \times m, k, \delta)$ observable codes. 
Definition 6 Let $\mathcal{C}$ be an $(n \times m, k, \delta)$ observable rank metric convolutional code. For $j \in \mathbb{N}_{0}$ we define the $j$-th column rank distance of $\mathcal{C}$ as

$$
d_{j}^{c r}=\min \left\{\operatorname{rwt}\left(V(D)_{\mid[0, j]}\right): V(D) \in \mathcal{C} \text { and } V_{0} \neq 0\right\},
$$

where for $V(D)=\sum_{i \in \mathbb{N}_{0}} V_{i} D^{i}$ we define $V(D)_{\mid[0, j]}=\sum_{i=0}^{j} V_{i} D^{i}$.

Theorem 7. Let $\mathcal{C}$ be an $(n \times m, k, \delta)$ observable rank metric convolutional code. Then the $j$-th column rank distance of $\mathcal{C}$ is upper bounded by

$$
d_{j}^{c r} \leq j\left(n-\left\lfloor\frac{k}{m}\right\rfloor\right)+n-\left\lfloor\frac{k-1}{m}\right\rfloor
$$

Proof. Let $G(D)=\sum_{i \in \mathbb{N}_{0}} G_{i} D^{i}$ be an encoder of $\mathcal{C}$. Since $G_{0}$ is full row rank it admits an invertible $k \times k$ submatrix. We can assume without loss of generality that the $k \times k$ submatrix of $G_{0}$ constituted by the first $k$ columns is invertible.

We will prove the theorem by induction on $j$. For $j=0$ let $u_{0} \in \mathbb{F}^{k}$ be such that $v_{0}=u_{0} G_{0}$ has the first $k-1$ entries equal to zero, i.e., $w t\left(v_{0}\right) \leq n m-k+1$, and let $V_{0}=\operatorname{rmat}_{n \times m}\left(v_{0}\right)$. Then the first $\left\lfloor\frac{k-1}{m}\right\rfloor$ rows of $V_{0}$ are equal to zero and therefore $\operatorname{rwt}\left(V_{0}\right) \leq n-\left\lfloor\frac{k-1}{m}\right\rfloor$ and therefore $d_{0}^{c r} \leq n-\left\lfloor\frac{k-1}{m}\right\rfloor$.

Let us suppose now that $d_{j}^{\text {cr }} \leq j\left(n-\left\lfloor\frac{k}{m}\right\rfloor\right)+n-\left\lfloor\frac{k-1}{m}\right\rfloor$ and let us prove that $d_{j+1}^{c r} \leq(j+1)\left(n-\left\lfloor\frac{k}{m}\right\rfloor\right)+n-\left\lfloor\frac{k-1}{m}\right\rfloor$. Let $u(D) \in \mathbb{F}[D]^{k}, v(D)=u(D) G(D)$ and $V(D)=\operatorname{rmat}_{n \times m}(v(D))=\sum_{i \in \mathbb{N}_{0}} V_{i} D^{i} \in \mathcal{C}$ be such that $\operatorname{rwt}\left(V(D)_{\mid[0, j]}\right)=d_{j}^{c r}$. Moreover, since the $k \times k$ submatrix of $G_{0}$ constituted by the first $k$ columns is invertible, we can consider $u_{j+1}$ such that $v_{j+1}=u_{j+1} G_{0}+u_{j-1} G_{1}+\cdots+u_{0} G_{j+1}$ has the first $k$ entries equal to zero. Then

$$
\begin{aligned}
d_{j+1}^{c r} & \leq \operatorname{rwt}\left((V(D))_{\mid[0, j+1]}\right) \\
& =d_{j}^{c r}+\operatorname{rwt}\left(V_{j+1}\right) \\
& \leq j\left(n-\left\lfloor\frac{k}{m}\right\rfloor\right)+n-\left\lfloor\frac{k-1}{m}\right\rfloor+n-\left\lfloor\frac{k}{m}\right\rfloor \\
& =(j+1)\left(n-\left\lfloor\frac{k}{m}\right\rfloor\right)+n-\left\lfloor\frac{k-1}{m}\right\rfloor .
\end{aligned}
$$

With a similar reasoning as in the proof of the above theorem we can prove that if the $j$-th column distance of a rank metric convolutional code achieves the corresponding bound then the same happens for all the $i$-th column distances for $i<j$.

Theorem 8. Let $\mathcal{C}$ be an $(n \times m, k, \delta)$ observable rank metric convolutional code. If $d_{j}^{c r}=j\left(n-\left\lfloor\frac{k}{m}\right\rfloor\right)+n-\left\lfloor\frac{k-1}{m}\right\rfloor$ for some $j \in \mathbb{N}_{0}$, then $d_{i}^{c r}=i\left(n-\left\lfloor\frac{k}{m}\right\rfloor\right)+$ $n-\left\lfloor\frac{k-1}{m}\right\rfloor$ for all $i \leq j$. 
Proof. It is enough to prove that $d_{j}^{c r}=j\left(n-\left\lfloor\frac{k}{m}\right\rfloor\right)+n-\left\lfloor\frac{k-1}{m}\right\rfloor$ implies that $d_{j-1}^{c r}=(j-1)\left(n-\left\lfloor\frac{k}{m}\right\rfloor\right)+n-\left\lfloor\frac{k-1}{m}\right\rfloor$. Let us assume that $d_{j-1}^{c r}<(j-1)\left(n-\frac{k}{m}\right)+$ $n-\left\lfloor\frac{k-1}{m}\right\rfloor$ and let $u(D) \in \mathbb{F}[D]^{k}, v(D)=u(D) G(D)$ and $V(D)=\operatorname{rmat}_{n \times m}(v(D))=$ $\sum_{i \in \mathbb{N}} V_{i} D^{i} \in \mathcal{C}$ be such $\operatorname{rwt}(V(D))_{\mid[0, j-1]}=d_{j-1}^{c r}$. Let $u_{j}$ be such that $v_{j}=$ $u_{0} G_{j}+u_{1} G_{j-1}+\cdots+u_{j-1} G_{1}+u_{j} G_{0}$ has weight $n m-k$. Then $\operatorname{rank}\left(V_{j}\right) \leq$ $n-\left\lfloor\frac{k}{m}\right\rfloor$ and, therefore, $w_{\text {rank }}\left(V(D)_{[0, j]}\right)<j\left(n-\left\lfloor\frac{k}{m}\right\rfloor\right)+n-\left\lfloor\frac{k-1}{m}\right\rfloor$. Consequently, $d_{j}^{c r}<j\left(n-\left\lfloor\frac{k}{m}\right\rfloor\right)+n-\left\lfloor\frac{k-1}{m}\right\rfloor$

It is obvious that the sequence of column rank distances of the code is nondecreasing. However, there exists an $M \in \mathbb{N}_{0}$ such that $d_{M}^{c r}=d_{j}^{c r}$ for $j>M$ since the column rank distances of a rank convolutional code can not be greater than the sum rank distance of the code. If the code is MRD then $M$ is precisely determined as stated in the next result.

Proposition 9 Let $\mathcal{C}$ be an $M R D(n \times m, k, \delta)$ observable rank metric convolutional code with column rank distances $d_{j}^{c r}, j \in \mathbb{N}_{0}$, and sum rank distance $d_{S R}$. Let $M=\min \left\{j \in \mathbb{N}_{0}, d_{j}^{c r}=d_{S R}\right\}$. Then,

$$
M=\left\lceil\frac{n\left\lfloor\frac{\delta}{k}\right\rfloor+\left\lfloor\frac{\delta-k\left\lfloor\frac{\delta}{k}\right\rfloor}{m}\right\rfloor}{n-\left\lfloor\frac{k}{m}\right\rfloor}\right\rceil
$$

Proof. Let $\tilde{M}=\frac{n\left\lfloor\frac{\delta}{k}\right\rfloor+\left\lfloor\frac{\delta-k\left\lfloor\frac{\delta}{k}\right\rfloor}{m}\right\rfloor}{n-\left\lfloor\frac{k}{m}\right\rfloor}$. We will consider two cases, when $m \mid k$ and when $m \nmid k$.

Case 1: $m \mid k$. Then

$$
\begin{aligned}
\tilde{M}\left(n-\left\lfloor\frac{k}{m}\right\rfloor\right)+n-\left\lfloor\frac{k-1}{m}\right\rfloor & =n\left\lfloor\frac{\delta}{k}\right\rfloor+\left\lfloor\frac{\delta-k\left\lfloor\frac{\delta}{k}\right\rfloor}{m}\right\rfloor+n-\left\lfloor\frac{k-1}{m}\right\rfloor \\
& =n\left(\left\lfloor\frac{\delta}{k}\right\rfloor+1\right)-\frac{k}{m}\left\lfloor\frac{\delta}{k}\right\rfloor+\left\lfloor\frac{\delta}{m}\right\rfloor-\left\lfloor\frac{k-1}{m}\right\rfloor .
\end{aligned}
$$

Then, since $\left\lfloor\frac{k-1}{m}\right\rfloor=\frac{k}{m}-1$, we have that

$$
\begin{aligned}
\tilde{M}\left(n-\left\lfloor\frac{k}{m}\right\rfloor\right)+n-\left\lfloor\frac{k-1}{m}\right\rfloor & =n\left(\left\lfloor\frac{\delta}{k}\right\rfloor+1\right)-\frac{k}{m}\left(\left\lfloor\frac{\delta}{k}\right\rfloor+1\right)+\left\lfloor\frac{\delta}{m}\right\rfloor+1 \\
& =n\left(\left\lfloor\frac{\delta}{k}\right\rfloor+1\right)-\left\lceil\frac{k\left(\left\lfloor\frac{\delta}{k}\right\rfloor+1\right)-\delta}{m}\right\rfloor+1 \\
& =d_{\mathrm{SR}} .
\end{aligned}
$$


Case 2: $m \nmid k$. In this case

$$
\begin{aligned}
\tilde{M}\left(n-\frac{k}{m}\right)+n-\left\lfloor\frac{k-1}{m}\right\rfloor & =n\left(\left\lfloor\frac{\delta}{k}\right\rfloor+1\right)+\left\lfloor\frac{\delta-k\left\lfloor\frac{\delta}{k}\right\rfloor}{m}\right\rfloor-\left\lfloor\frac{k-1}{m}\right\rfloor \\
& =n\left(\left\lfloor\frac{\delta}{k}\right\rfloor+1\right)-\left\lceil\frac{k\left(\left\lfloor\frac{\delta}{k}\right\rfloor+1\right)-\delta-k}{m}\right\rfloor-\left\lfloor\frac{k-1}{m}\right\rfloor \\
& =n\left(\left\lfloor\frac{\delta}{k}\right\rfloor+1\right)-\left(\left\lfloor\frac{k\left(\left\lfloor\frac{\delta}{k}\right\rfloor+1\right)-\delta}{m}\right\rfloor-\left\lfloor\frac{k}{m}\right\rfloor\right)-\left\lfloor\frac{k-1}{m}\right\rfloor \\
& =n\left(\left\lfloor\frac{\delta}{k}\right\rfloor+1\right)-\left\lceil\frac{k\left(\left\lfloor\frac{\delta}{k}\right\rfloor+1\right)-\delta}{m}\right\rfloor+1 \\
& =d_{\mathrm{SR}},
\end{aligned}
$$

because $\left\lfloor\frac{k}{m}\right\rfloor-\left\lfloor\frac{k-1}{m}\right\rfloor=1$.

In both cases $M=\lceil\tilde{M}\rceil$.

\section{References}

1. A. Badr, A. Khisti, Wai-Tian. Tan, and J. Apostolopoulos. Layered constructions for low-delay streaming codes. IEEE Trans. Inform. Theory, 2013 submitted.

2. J.J. Climent, D. Napp, C. Perea, and R. Pinto. Maximum distance separable 2D convolutional codes. IEEE Trans. Information Theory, 62(2):669-680, 2016.

3. H. Gluesing-Luerssen, J. Rosenthal, and R. Smarandache. Strongly MDS convolutional codes. IEEE Trans. Inform. Theory, 52(2):584-598, 2006.

4. R. Johannesson and K. Sh. Zigangirov. Fundamentals of Convolutional Coding. IEEE Press, New York, 1999.

5. R. Kötter and F.R. Kschischang. Coding for errors and erasures in random network coding. IEEE Trans. Inform. Theory, 54(8):3579-3591, Aug 2008.

6. R. Mahmood. Rank metric convolutional codes with applications in network streaming. Master of applied science, 2015.

7. R. Mahmood, A. Badr, and A. Khisti. Streaming-codes for multicast over burst erasure channels. IEEE Trans. Inform. Theory, 61(8):4181-4208, 2015.

8. R. Mahmood, A. Badr, and A. Khisti. Convolutional codes with maximum column sum rank for network streaming. IEEE Trans. Inform. Theory, 62(6):3039-3052, 2016.

9. R. J. McEliece. The algebraic theory of convolutional codes. In V. Pless and W.C. Huffman, editors, Handbook of Coding Theory, volume 1, pages 1065-1138. Elsevier Science Publishers, Amsterdam, The Netherlands, 1998.

10. R.J. McEliece. The algebraic theory of convolutional codes. In R.A. Brualdi V.S. Pless, W.C. Huffman, editor, Handbook of Coding Theory Vol. 1. NorthHolland, Amsterdam, 1998.

11. D. Napp, R. Pinto, J. Rosenthal, and P. Vettori. Rank metric convolutional codes. Proceedings of the 22nd International Symposium on Mathematical Theory of Network and Systems (MTNS), Minnesota, USA, 2016.

12. D. Napp, R. Pinto, J. Rosenthal, and P. Vettori. MRD rank metric convolutional codes. IEEE International Symposium on Information Theory (ISIT) 2017, 2017. 
13. D. Napp, R. Pinto, and V. R. Sidorenko. Concatenation of convolutional codes and rank metric codes for multi-shot network coding. submitted to Des. Codes Cryptogr.

14. D. Napp, R. Pinto, and M. Toste. On MDS convolutional codes over $\mathbb{Z}_{p^{r}}$. Designs, Codes and Cryptography, 83:101-114, 2017.

15. R.W. Nóbrega and B.F. Uchoa-Filho. Multishot codes for network coding using rank-metric codes. In Wireless Network Coding Conference (WiNC), 2010 IEEE, pages 1-6, June 2010.

16. J. Rosenthal and R. Smarandache. Maximum distance separable convolutional codes. Appl. Algebra Engrg. Comm. Comput., 10(1):15-32, 1999.

17. A. Wachter-Zeh, M. Stinner, and V. Sidorenko. Convolutional codes in rank metric with application to random network coding. IEEE Trans. Inform. Theory, 61(6):3199-3213, June 2015. 